\title{
S-G-2. Studies on NGF (Nerve Growth Promoting Factor)
}

\author{
- Control of Nerve Regeneration by Specific \\ Protein and its Antiserum-- \\ Tsuneyuki NAKAzAWA \\ Dept. of Neuropsychiatry, Keio University
}

\section{S-G-3. Possibility of Anastomosis of the Spinal Cord}

\author{
Hiroaki Kawakita, Kazuo Nishimoto, Sadahiko Maruno, \\ Haruki Kondo, Kyo Fujimoto, Akira Ito, \\ Norio OONISHI and Kiyoto TAKAO \\ Dept. of Surgery, Kobe University School of Medicine
}

\section{S-G-4. Regeneration of Peripheral Nerves by Irradiated Homografts}

\author{
Naoki Kageyama, Kimiyuki IKedA, Shoji NaKaJIma, \\ Morio Matsunaga and Hajime Handa \\ Department of Neurosurgery, Kyoto University Medical School
}

We have previously reported that peripheral nerve of rats or dogs can be successfully homografted if they were irradiated with 2 million REP of electron beam for the purpose of suppressing the immune reaction. Histological and electrophysiological analyses are hereby attempted on fourteen cases which we have successfully kept alive for long-term observation. In addition, availability of vascular tubulation technique to nerve anastomosis and importance of generalized immune suppressive treatment in cases of nerve homografting are discussed.

In twelve successful cases out of fourteen, which we had followed up for more than 180 days after surgery, regenerated nerves were found to penetrate through the graft down into the distal stump mostly with fasciculus formation. Axonal counts at the proximal stump, the grafted portion and the distal stump performed with two dogs, which were sacrificed at 203 rd 